\title{
The effect of osteoprotegerin administration on the intra-tibial growth of the osteoblastic LuCaP 23.1 prostate cancer xenograft
}

\author{
J.A. Kiefer ${ }^{1}$, R.L. Vessella ${ }^{1,2}$, J.E. Quinn ${ }^{1}$, A.M. Odman ${ }^{1}$, J. Zhang ${ }^{3}$, E.T. Keller ${ }^{3}$, P.J. Kostenuik ${ }^{4}$, C.R. \\ Dunstan $^{4} \&$ E. Corey ${ }^{1}$ \\ ${ }^{1}$ Department of Urology, University of Washington School of Medicine, Seattle, Washington, USA; ${ }^{2}$ VA Medical \\ Center, Seattle, Washington USA; ${ }^{3}$ Department of Pathology, University of Michigan, Ann Arbor, Michigan, \\ USA; ${ }^{4}$ Amgen, Inc., Thousand Oaks, California, USA
}

Received 30 May 2003; accepted in revised form 10 May 2004

Key words: bone metastases, bone remodeling, osteoblast, osteoprotegerin, prostate cancer, PSA

\begin{abstract}
Osteoprotegerin (OPG) plays a central role in controlling bone resorption. Exogenous administration of OPG has been shown to be effective in preventing osteolysis and limiting the growth of osteolytic metastasis. The objective of this study was to investigate the effects of OPG on osteoblastic prostate cancer $(\mathrm{CaP})$ metastases in an animal model. LuCaP 23.1 cells were injected intra-tibially and Fc-OPG $(6.0 \mathrm{mg} / \mathrm{kg})$ was administered subcutaneously three times a week starting either 24 hours prior to cell injection (prevention regimen) or at 4 weeks post-injection (treatment regimen). Changes in bone mineral density at the tumor site were determined by dual x-ray absorptiometry. Tumor growth was monitored by evaluating serum prostate specific antigen (PSA). FcOPG did not inhibit establishment of osteoblastic bone lesions of $\mathrm{LuCaP} 23.1$, but it decreased growth of the tumor cells, as determined by decreases in serum PSA levels of $73.0 \pm 44.3 \%(P<0.001)$ and $78.3 \pm 25.3 \%$ $(P<0.001)$ under the treatment and prevention regimens, respectively, compared to the untreated tumor-bearing animals. Administration of Fc-OPG decreased the proliferative index by $35.0 \%(P=0.1838)$ in the treatment group, and $75.2 \%(P=0.0358)$ in the prevention group. The results of this study suggest a potential role for OPG in the treatment of established osteoblastic $\mathrm{CaP}$ bone metastases.
\end{abstract}

Abbreviations: bone mineral density - BMD; 3,3'-diaminobenzidine tetrahydrochloride - DABC; prostate cancer - CaP; dual x-ray absorptiometry - DEXA; osteoprotegerin - OPG; prostate specific antigen - PSA; receptor activator of NF- $\kappa \mathrm{B}-\mathrm{RANK}$; receptor activator of NF- $\kappa \mathrm{B}$ ligand $-\mathrm{RANKL}$

\section{Introduction}

A characteristic property of metastatic prostate cancer $(\mathrm{CaP})$ is its propensity to colonize and thrive in skeletal tissue. CaP skeletal metastases are associated with advanced disease and represent a significant therapeutic challenge. Skeletal deposits of metastatic $\mathrm{CaP}$ alter the normal physiology of the bone, leading to disruption of the bone remodeling cycles. Normal remodeling is marked by the coordinated activities of bone formation and resorption, which are essential for the proper function and integrity of skeletal tissue [1]. CaP disruption of bone remodeling usually causes a net increase in bone formation leading to the development of osteoblastic lesions [2, 3];

Correspondence to: Eva Corey, PhD, Department of Urology, Mailstop 356510, University of Washington, 1959 NE Pacific St. HSB I-340, Seattle, WA 98195, USA.

Tel: + 1-206-543-1461; Fax: + 1-206-543-1146;

E-mail: ecorey@u.washington.edu however, a significant resorptive component is also observed [4-7]. Release of growth factors from the mineralized matrix during resorption may facilitate the initial seeding and growth of tumor cells in the bone [8]. Many of the proteins involved in these processes have now been identified, and the alterations in bone remodeling observed in $\mathrm{CaP}$ bone metastases offer an increasingly attractive target for therapeutic intervention.

A key molecular system that influences bone remodeling consists of receptor activator of NF- $\kappa \mathrm{B}$ ligand (RANKL), receptor activator of NF- $\kappa$ B (RANK), and osteoprotegerin (OPG). OPG is a member of the tumor necrosis factor receptor superfamily that functions as a decoy receptor for RANKL. Association of RANKL with OPG prevents it from interacting with its cognate receptor, RANK. An increase in the ratio of OPG to RANKL inhibits the recruitment, activation and survival of osteoclasts and results in increased bone mass. Transgenic mice overexpressing OPG exhibit an 
osteopetrotic phenotype [9], while OPG-knockout mice have severe osteoporosis [10, 11]. Thus the OPG/ RANKL ratio is an important element in the control of normal bone remodeling.

The RANKL/RANK/OPG system is also involved in various pathologies associated with metastatic disease in bone [12]. An elevated RANKL/OPG ratio is observed in osteolytic malignancies such as breast cancer and multiple myeloma [13-17] and we have recently reported increased expression of RANKL in $\mathrm{CaP}$ bone metastases [18]. Treatment with Fc-OPG in certain mouse models diminishes growth and osteolytic lesions of myeloma and breast cancer cells in bone [17, 19]. In prostate cancer, Fc-OPG administration decreased the establishment and subsequent intra-osseous growth of $\mathrm{C} 4-2 \mathrm{~B} \mathrm{CaP}$ cells, which stimulate osteoclast formation in vitro [20], and $\mathrm{LNCaP}$ cells, which give rise to mixed osteoblastic/osteolytic lesions [21]. These results suggest that osteoclast activation may play an important role in promoting the initial growth of $\mathrm{CaP}$ in the bone environment.

In this study we have used the $\mathrm{CaP}$ xenograft $\mathrm{LuCaP}$ 23.1, which induces production of new bone, resulting in sclerotic lesions in murine models. Administration of $\mathrm{Fc}-\mathrm{OPG}$ inhibited the growth of $\mathrm{LuCaP}$ 23.1 xenograft in the bone, but did not alter the establishment of skeletal lesions. The results suggest potential benefits of OPG in the treatment of patients with established osteoblastic $\mathrm{CaP}$ bone metastases.

\section{Methods}

\section{Animal studies}

All animal experiments were performed in compliance with the University of Washington Institutional Animal Care and Use Committee and NIH guidelines. The LuCaP 23.1 xenograft was established in our laboratory from a pelvic lymph node metastasis [22, 23]. It elicits a robust osteoblastic reaction in bone [24]. Single-cell suspensions of $\mathrm{LuCaP} 23.1$ cells were used for direct intra-tibial injections as we have previously described [24].

Thirty-six-week-old Fox Chase SCID male mice (Charles River, Wilmington, Massachusetts) were injected with $1-2 \times 10^{5}$ cells into the right tibiae. The animals were then randomized into three groups: 1) a control group $(n=10) ; 2)$ a treatment group $(n=$ 10 ), with Fc-OPG injections beginning 4 weeks after xenograft injections; and 3) a prevention group ( $n=$ 10), with Fc-OPG injections beginning $24 \mathrm{~h}$ prior to injection of tumor cells. The Fc-OPG used in this study contains the ligand-binding domain of human OPG fused to the $\mathrm{Fc}$ domain of human $\operatorname{IgG}[9,19]$. Fc-OPG $(6.0 \mathrm{mg} / \mathrm{kg})$ was administered subcutaneously three times a week; the control group received injections of vehicle (PBS). Tumor burden was monitored during the study by measuring serum levels of prostate specific antigen (PSA). Determination of serum PSA was performed weekly starting at day 16 (IMx Total PSA Immunoassay, Abbot Laboratories, Abbott Park, Illinois). Mice were sacrificed 8 weeks after the xenograft injection. The time of sacrifice was assigned based on serum PSA levels of the control group and the radiographical appearance of the tumored tibiae (indicating mid-stage LuCaP 23.1 osseous tumors). Prior to sacrifice, all mice were radiographed with a diagnostic mammography unit (LoRad, Danbury, Connecticut) with the following settings: $35 \mathrm{kv}$, $2.3 \mathrm{sec}, 20.7 \mathrm{~mA} / \mathrm{sec}$. Both tumor-bearing and contralateral tibiae were harvested for bone mineral density (BMD) determinations. Tumor-bearing tibiae were then processed for immunohistochemical analysis.

\section{Bone densitometry}

BMD was measured using dual-energy X-ray absorptiometry (DEXA) on an Eclipse peripheral DEXA Scanner (Norland, Ft. Atkinson, Wisconsin). Data analysis was performed with software provided by the company. The region of interest $(2.5 \mathrm{~mm} \times 2.5 \mathrm{~mm})$ encompassed the tumor injection site and was located $2.0 \mathrm{~mm}$ from the proximal end of the tibiae. Excised tibiae were placed laterally on the scanner for analysis. Scanning was done at $2 \mathrm{~mm} / \mathrm{s}$ with a resolution of $0.1 \mathrm{~mm} \times 0.1 \mathrm{~mm}$. Short-term BMD precision $(\%$ coefficient of variation) was less than $3 \%$ for this technique.

\section{Immunohistochemistry}

Tibiae with LuCaP 23.1 tumors were processed for histology and immunohistochemistry by fixation in $10 \%$ neutral buffered formalin for 24 hours. After fixation, tibiae were decalcified in $10 \%$ EDTA for two consecutive 48-hour periods and embedded in paraffin. $5.0-\mu \mathrm{m}$ longitudinal sections were used for staining. Histological verification of intra-tibial tumors was assessed by hematoxylin and eosin (H\&E) staining. Tibial sections were also examined immunohistochemically for expression of PSA and human Ki-67. Briefly, sections were deparaffinized, rehydrated, and incubated for $10 \mathrm{~min}$ at room temperature in $3 \% \mathrm{H}_{2} \mathrm{O}_{2}$ in methanol to block endogenous peroxidase activity. Specimens were blocked for one hour in a cocktail of 5\% horse, $5 \%$ goat, and $5 \%$ chicken serum in $1 \times$ PBS. The sections for Ki-67 staining underwent antigen retrieval in $1 \times$ citrate buffer ( $\mathrm{pH}$ 6.0) for $10 \mathrm{~min}$. Detection of PSA was performed using a rabbit polyclonal anti-PSA antibody (DAKO, Carpinteria, California), diluted in blocking cocktail, at a concentration of $3 \mu \mathrm{g} / \mathrm{ml}$ for one hour at room temperature. A rabbit polyclonal anit-Ki-67 (DAKO) was used at a dilution of 1:50. Normal rabbit IgG (DAKO) served as the negative control. After incubation of primary antibodies and subsequent washing, sections were stained with biotinylated goat anti-rabbit IgG (DAKO) at a dilution of 
1:150 in blocking cocktail for one hour at room temperature. Immunoreactiviy was detected with the Vectastain Elite ABC kit (Vector Laboratories, Burlingame, California) and color-developed with 3,3'diaminobenzidine tetrahydrochloride (DABC). The proliferative index was determined by counting Ki-67positive nuclei. In every sample, three representative 40x-fields were scored for positive tumor nuclei $(85$ nuclei on average; control group and prevention group $n=3$ animals, treatment group $n=2$ animals).

\section{Statistical analysis}

Statistical analysis was performed using GraphPad Prism (GraphPad Software, Inc., San Diego, California). Significance of the differences between the groups was determined using a two-tailed Student's $t$-test. Association between PSA levels and proliferative index was evaluated using the Pearson correlation.

\section{Results}

LuCaP 23.1 xenograft grew in the tibiae of SCID male mice and yielded an osteoblastic reaction. Skeletal LuCaP 23.1 lesions were established in $100 \%$ of the animals in the control and prevention groups and $90 \%$ in the treatment group. Three mice from the control group, two from the treatment group, and one mouse from the prevention group were excluded from analysis due to compromised integrity of the tibial shaft resulting from tumor delivery, and subsequent growth of tumor in muscle. One mouse in the prevention group expired during the study and was excluded from subsequent analysis. Evidence of increased bone mass is observed in the $\mathrm{x}$-rays of the tumor-bearing tibiae (Figure 1A). A large volume of new trabecular bone mass was observed in tumor-bearing tibiae of all three groups, but it was most noticeable in the prevention and treatment groups (Figure 1). Tumor foci were present in the tibia in all three groups; however, in the groups that received Fc-OPG, tumor foci decreased in size (Figure 1).

\section{Bone mineral density}

Representative examples of DEXA scans and the effects of Fc-OPG administration on the BMD of Lu$\mathrm{CaP}$ 23.1-containing and contralateral non-tumorbearing tibiae are shown in Figure 2. In accordance with its osteoblastic character, LuCaP 23.1 caused significant increases in BMD over the contralateral nontumor-bearing tibiae $(P=0.0004)$ of the control group. Fc-OPG administration in the treatment group tumor-bearing tibiae did not significantly alter BMD vs LuCaP 23.1-bearing tibiae of the control group $(P=0.2767)$, but longer administration ( 8 weeks) of Fc-OPG in the prevention group increased BMD

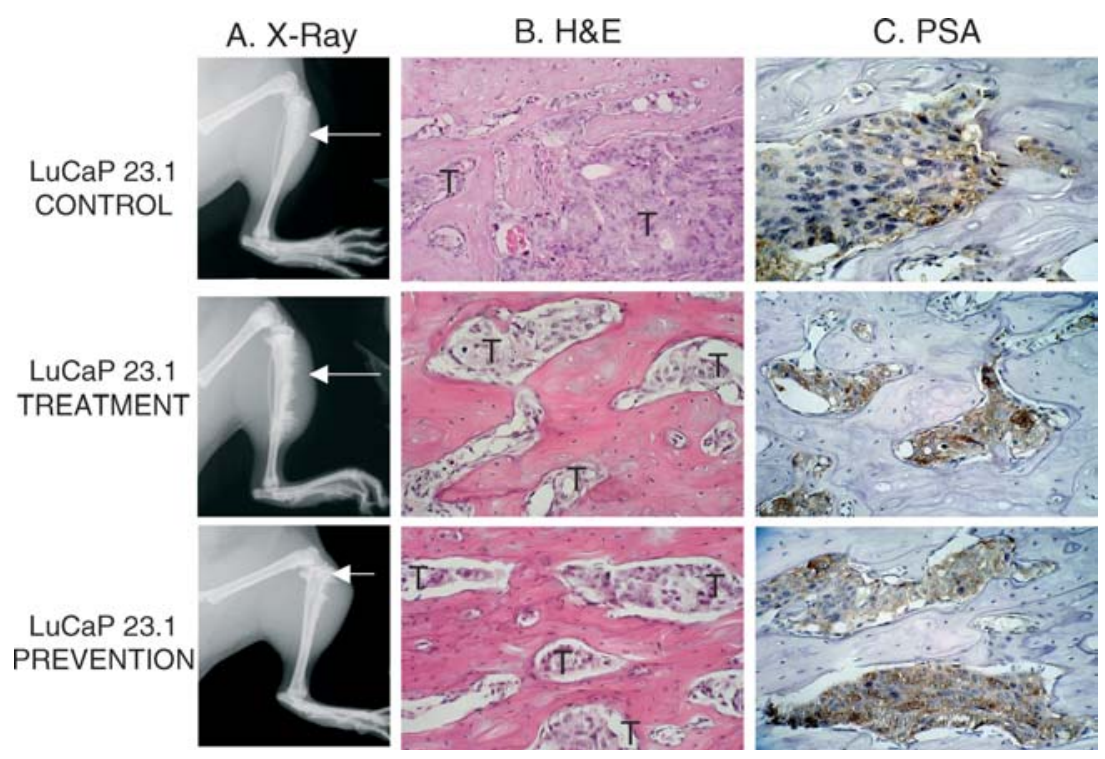

Figure 1. Representative photomicrographs of LuCaP 23.1 bone metastases with and without administration of Fc-OPG. Tibiae of SCID mice were injected with LuCaP 23.1 cells and Fc-OPG was administered under prevention and treatment regimens as described in Methods. (A) Representative radiographs of LuCaP 23.1 tumors in tibiae. Radiographs were taken using a mammography unit. LuCaP 23.1 cells caused a strong osteoblastic reaction in the bone in all three groups (control, prevention, treatment) as indicated by arrow. The tibiae containing tumor are wider and denser, as represented by whiter color in the radiographs. Spiking of outgrowing bone caused by the tumor is seen in the upper right and lower portions of the preventive-group tibia. In the portion between these spikes the original cortical shaft has been conserved. (B) Representative histology of LuCaP 23.1 tumors in tibiae. Five-micrometer sections of decalcified LuCaP 23.1 tibiae were stained with hematoxilin \& eosin. Tumor foci (designated by a T) are observed in samples from all three groups. Large trabecular bone mass is observed in all groups. (C) Represenative PSA staining of LuCaP 23.1 tumors in tibiae. Five-micrometer sections of decalcified LuCaP 23.1 tibiae were stained with antiPSA antibody. PSA-positive cells were detected in tibiae from all three groups. Intensive PSA immunoreactivity is observed in tumors from all three groups. 
(a)

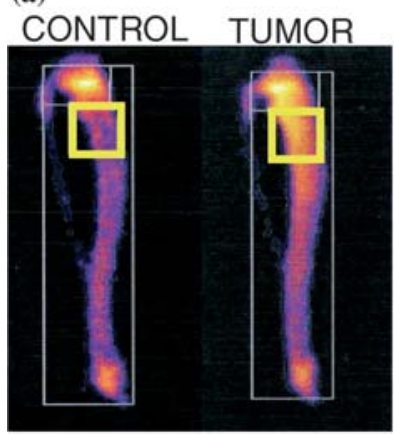

(b)

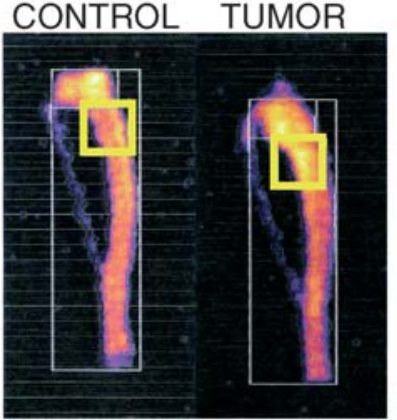

(c)

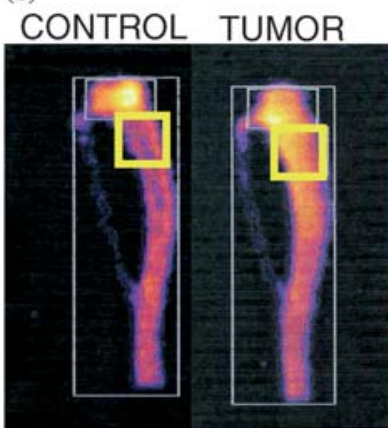

(d)

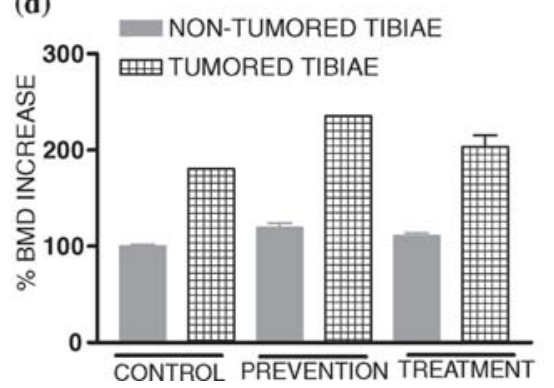

Figure 2. Effects of Fc-OPG on bone mineral density of tibiae with osteoblastic LuCaP 23.1 metastases. (a) Representative examples of DEXA scans of LuCaP 23.1 bone metastases with or without Fc-OPG administration: (a) control group, (b) prevention group, (c) treatment group. Yellow color indicates areas of high BMD. The xenograft injection site is marked by yellow box. (d) BMD was measured at the site of injection of tumor cells as described in Methods. Results were normalized to non-tumor-bearing tibiae of untreated animals, and are presented as mean \pm SEM. Significant increases of BMD were observed in the control group tumor-bearing tibiae vs contralateral non-tumored tibiae $(P=$ 0.0004). Administration of Fc-OPG caused additional increases in BMD of LuCaP 23.1 tibiae (treatment: $P<0.0001$, prevention: $P<0.0001$ ) when compared to non-tumor bearing contralateral tibia. Fc-OPG administration under both regimens also caused increases in BMD in nontumor bearing tibiae vs. control non-tumored tibiae (treatment: $P=0.0081$, prevention: $P=0.0026$ ). LuCaP 23.1 causes an osteoblastic reaction in the bone; therefore one may expect that a smaller tumor burden should result in decreased BMD. However, since OPG is a negative regulator of bone lysis, administration of Fc-OPG also causes increases in BMD. (There are two opposing effects of Fc-OPG on BMD: inhibition of tumor growth, which tends to reduce BMD, and inhibition of osteolysis, which increases BMD.) Since we have shown that Fc-OPG inhibits proliferation of LuCaP 23.1 in bone, we attributed the increases in BMD to inhibition of osteolysis by Fc-OPG. The significance of the differences was determined by the Student's $t$-test.

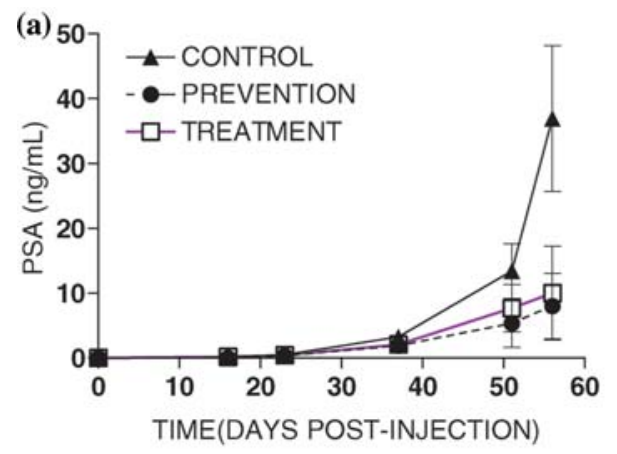

(b)
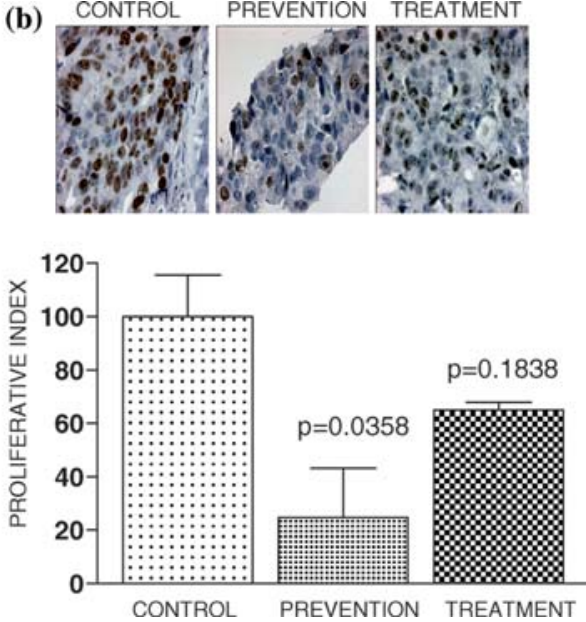

Figure 3. (a) Effects of Fc-OPG on serum PSA levels in animals bearing LuCaP 23.1 bone metastases. Blood samples were drawn from animals with $\mathrm{LuCaP} 23.1$ bone metastases weekly starting at day 16 after injection of the tumor cells. PSA levels were determined by IMx Total PSA assay; mean \pm SEM is plotted against time post-injection. Significant decreases in serum levels of PSA were detected at day 57 in both prevention $(78.3 \pm 25.3 \%$ decrease, $P<0.001)$ and treatment $(73.0 \pm 44.3 \%$ decrease, $P<0.001)$ groups vs control group. (b) Effects of Fc-OPG on proliferation of LuCaP 23.1 cells in the bone environment. Five-micrometer sections of decalcified LuCaP 23.1 tibiae were stained with anti-Ki-67 antibody. Representative examples of Ki67 immunoreactivity and analysis of the results are presented. Data were normalized to control group and are shown as a percent decrease in positive nuclei. A decrease in the proliferation index of $35.0 \%(P=0.1838)$ in the treatment group, and $75.2 \%(P=0.0358)$ was detected in the prevention group. 
significantly $(P=0.0393)$ over the BMD of the Lu$\mathrm{CaP} 23.1$ tibiae of the control group. Fc-OPG administration under both regimens significantly increased BMD in non-tumor-bearing tibiae $(P=0.0081, P=$ 0.0026; prevention and treatment, respectively). LuCaP 23.1 causes an osteoblastic reaction in the bone; therefore a smaller tumor burden should lead to lower osteoblast activity and decreased BMD. However, since OPG is a negative regulator of bone lysis, administration of $\mathrm{Fc}-\mathrm{OPG}$ also causes an increase in BMD. There are two opposing effects of Fc-OPG on BMD - inhibition of tumor growth, which tends to reduce $\mathrm{BMD}$, and inhibition of osteolysis, which increases BMD. Since we have shown that Fc-OPG inhibits proliferation of $\mathrm{LuCaP} 23.1$ in bone, we attribute an observed increase in BMD in the prevention group vs the control to the second effect of $\mathrm{Fc}-\mathrm{OPG}$, inhibition of osteolysis.

\section{Serum PSA levels}

To monitor tumor burden in the tibiae, serum PSA levels were measured in animals bearing LuCaP 23.1. There was a trend toward reduced serum PSA levels in the prevention and treatment groups, compared to the control group, over the course of the study (Figure 3A). At day 56 post-injection, there were significant decreases in PSA levels in both treatment $(73.0 \pm$ $44.3 \%, \quad P<0.001)$ and prevention $(78.3 \pm 25.3 \%$, $P<0.001)$ groups vs control group. Serum PSA levels are used to detect $\mathrm{CaP}$ and monitor the response of the disease to the treatment. However, in some cases serum PSA levels in patients are not always representative of the tumor burden, since various factors present in bone (e.g., IL-6) can affect PSA expression. We have previously shown that the tumor volume of $\mathrm{LuCaP} 23.1$ is correlated with serum PSA levels in animals bearing Lu$\mathrm{CaP} 23.1$ subcutaneous tumors as well as tumors in tibiae [22-25]. Therefore in the present study we conclude that significantly lower levels of PSA in serum of treated animals suggest decreased tumor burden of osteoblastic $\mathrm{LuCaP} 23.1$ in bone.

\section{Proliferation index}

Reduced serum PSA levels in animals treated with Fc-OPG suggest a relative reduction in tumor burden $[24,25]$. We measured the proliferation index of the $\mathrm{LuCaP} 23.1$ xenografts in tibiae to determine whether reduced PSA levels were associated with a decrease in proliferation of tumor cells in tibiae. FcOPG administration under both regimens decreased the proliferative index of $\mathrm{LuCaP} 23.1$ in tibiae (Figure 3b). The proliferation index of $\mathrm{LuCaP} 23.1$ decreased by $35.0 \%(P=0.1838)$ in the treatment group and $75.2 \%(P=0.0358)$ in the prevention group. A significant correlation was observed between the proliferative index and serum PSA level (Pearson $r=0.771, P=0.0254$ ).

\section{Discussion}

The majority of $\mathrm{CaP}$ bone metastases exhibit osteoblastic characteristics, and animal models that possess this trait are therefore of special interest for the study of human disease. However, despite the overall osteoblastic appearance of many $\mathrm{CaP}$ bone metastases, an osteolytic component is frequently evident within these lesions. It has been hypothesized that the increased rates of bone-formation and -resorption cycles associated with $\mathrm{CaP}$ metastases may promote skeletal metastases $[11,26]$. These observations and ideas give rise to the paradox that osteoblastic $\mathrm{CaP}$ metastases may be successfully treated by inhibition of osteolysis. In the present study we have sought to investigate this hypothesis by administering the antiresorptive molecule OPG to the LuCaP 23.1 osteoblastic xenograft injected into the tibiae of SCID mice.

Administration of Fc-OPG attenuated LuCaP 23.1 skeletal tumor growth, as indicated by reduced serum PSA levels and a decreased LuCaP 23.1 proliferation index. These observations suggest that normal and/or pathological bone resorption may promote the growth of $\mathrm{LuCaP} 23.1$ cells in bone, and that inhibition of osteolysis blocks or attenuates the growth process. We have reported previously [25] that $\mathrm{LuCaP} 23.1$ in the bone environment causes a decrease in the number of osteoclasts vs normal tibia. We have also shown separately that administration of the osteolysis-inhibiting compounds zoledronic acid [25] and Fc-OPG (our unpublished data) causes a trend toward further decreases in osteoclast numbers in the $\mathrm{LuCaP} 23.1$ model of bone metastasis; however, because $\mathrm{LuCaP}$ 23.1 itself inhibits osteoclast formation, the cell numbers involved are very small even without the treatment, and these further decreases generally do not achieve statistical significance.

Inhibition of bone resorption by OPG may also lead to a reduction in available growth-promoting substances liberated from the bone matrix by osteoclast. It is possible that reduction in bone lysis with Fc-OPG treatment reduced the growth of tumor cells by mechanisms related to inhibition of osteolysis. However, another therapeutic mechanism of action might involve direct effects of Fc-OPG on tumor cells. Work from other laboratories [11, 19] indicates that Fc-OPG fails to inhibit the growth of various tumor cells in vitro. This is not surprising, as there are no known signaling receptors for OPG. Nevertheless, OPG may affect tumor cells by blocking RANKL-mediated signals. Cells of the osteoclast lineage, along with breast epithelium and dendritic cells, respond to RANKL signaling, leading to differentiation and survival [27], and inhibition of this signaling pathway may slow the growth of $\mathrm{CaP}$ cells directly. Experimental confirmation of this hypothesis is not possible with the $\mathrm{LuCaP} 23.1$ xenograft because it does not grow in vitro. 
In contrast to the observed inhibition of growth of LuCaP 23.1 bone metastases, the rate of establishment of LuCaP 23.1 in the bone was not altered by administration of Fc-OPG. Similarly, we have reported previously that zoledronic acid, another anti-resorptive compound, did not affect the establishment of LuCaP 23.1 growth in the tibia [25]. Lee et al. also failed to observe inhibitory effects of zoledronic acid on tumor establishment of the osteoblastic CaP xenograft LAPC9 in bone [28]. These results contrast with those of an earlier study in which Fc-OPG inhibited the establishment of C4$2 \mathrm{~B}$ in the tibiae of SCID mice. The particular properties of the tumor models used in the studies may affect the results. In the present study we have used the LuCaP 23.1 xenograft, which yields osteoblastic lesions [24]. Zhang et al. used C4-2B cells [20], and Yonou et al. used LNCaP [21], both of which result in mixed lytic/blastic lesions. As with C4-2B, Fc-OPG administration has been shown to inhibit establishment of lytic bone metastases of myleoma and breast [17, 19]. Therefore stimulation of osteolysis by tumor cells appears to be a critical factor in the colonization of bone by tumors that exhibit mixed or osteolytic phenotypes.

Previous animal studies have shown that treatment with Fc-OPG results in a generalized increase in overall bone density [9]. Administration of FcOPG for four weeks did not significantly change the BMD of the tumor-bearing tibiae under the treatment regimen vs the untreated LuCaP 23.1 tibiae, although it did significantly increase the BMD in the treated non-tumor-bearing tibiae vs the control nontumor-bearing tibiae. The observed decrease in serum PSA levels and proliferative rate indicates a lower tumor burden in the treatment group. One would therefore expect lower levels of BMD vs the control untreated tumor-bearing tibiae. We hypothesize that the administration of Fc-OPG, in conjunction with an initial LuCaP 23.1 growth period, increased the BMD of the tumored tibiae vs non-tumor-bearing tibiae of the control group.

In conclusion, our results show that administration of Fc-OPG attenuated the growth of the LuCaP 23.1 tumor in the bone environment as determined by serum PSA levels and decreased proliferative index, but it did not inhibit the initial establishment of LuCaP 23.1 osteoblastic bone metastases. While results of this study do not delineate the mechanisms of growth inhibition of osseous $\mathrm{CaP}$ metastasis by OPG, they indicate the potential value of employing exogenous Fc-OPG in combination with other therapeutics in the treatment of established CaP-osseous metastasis.

\section{Acknowledgements}

We wish to thank Dr Michael Corey for editorial assistance, and Lisha Brown and Austin Odman for excellent technical assistance. Amgen, Inc. kindly provided Fc-OPG, and Abbott Laboratories reagents for determination of PSA serum levels. This research was supported by the Richard M. Lucas Cancer Foundation, and PO1 CA85859-01A.

\section{References}

1. Heaney RP. The bone-remodeling transient: Implications for the interpretation of clinical studies of bone mass change. J Bone Miner Res 1994; 9(10): 1515-23.

2. Clarke NW, McClure J, George NJ. The effects of orchidectomy on skeletal metabolism in metastatic prostate cancer. Scand J Urol Nephrol 1993; 27(4): 475-83.

3. Goltzman D. Mechanisms of the development of osteoblastic metastases. Cancer 1997; 80(8 Suppl): 1581-7.

4. Charhon SA, Chapuy MC, Delvin EE et al. Histomorphometric analysis of sclerotic bone metastases from prostatic carcinoma special reference to osteomalacia. Cancer 1983; 51(5): 918-24.

5. Urwin GH, Percival RC, Harris $\mathrm{S}$ et al. Generalised increase in bone resorption in carcinoma of the prostate. Br J Urol 1985; 57(6): 721-3.

6. Percival RC, Urwin GH, Harris S et al. Biochemical and histological evidence that carcinoma of the prostate is associated with increased bone resorption. Eur J Surg Oncol 1987; 13(1): $41-9$.

7. Clarke NW, McClure J, George NJ. Morphometric evidence for bone resorption and replacement in prostate cancer. $\mathrm{Br} \mathbf{J}$ Urol 1991; 68(1): 74-80.

8. Hauschka PV, Mavrakos AE, Iafrati MD et al. Growth factors in bone matrix. Isolation of multiple types by affinity chromatography on heparin-Sepharose. J Biol Chem 1986; 261(27): 12665-74.

9. Simonet WS, Lacey DL, Dunstan CR et al. Osteoprotegerin: A novel secreted protein involved in the regulation of bone density. Cell 1997; 89(2): 309-19.

10. Mizuno A, Amizuka N, Irie K et al. Severe osteoporosis in mice lacking osteoclastogenesis inhibitory factor/osteoprotegerin. Biochem Biophys Res Commun 1998; 247(3): 610-5.

11. Bucay N, Sarosi I, Dunstan CR et al. Osteoprotegerin-deficient mice develop early onset osteoporosis and arterial calcification. Genes Dev 1998; 12(9): 1260-8.

12. Hofbauer LC, Neubauer A, Heufelder AE. Receptor activator of nuclear factor-kappaB ligand and osteoprotegerin: Potential implications for the pathogenesis and treatment of malignant bone diseases. Cancer 2001; 92(3): 460-70.

13. Thomas RJ, Guise TA, Yin JJ et al. Breast cancer cells interact with osteoblasts to support osteoclast formation. Endocrinology 1999; 140(10): 4451-8.

14. Roux S, Amazit L, Meduri G et al. RANK (receptor activator of nuclear factor kappa B) and RANK ligand are expressed in giant cell tumors of bone. Am J Clin Pathol 2002; 117(2): 210-6.

15. Kitazawa S, Kitazawa R. RANK ligand is a prerequisite for cancer-associated osteolytic lesions. J Pathol 2002; 198(2): 22836.

16. Mancino AT, Klimberg VS, Yamamoto $M$ et al. Breast cancer increases osteoclastogenesis by secreting M-CSF and upregulating RANKL in stromal cells. J Surg Res 2001; 100(1): 18-24.

17. Croucher PI, Shipman CM, Lippitt J et al. Osteoprotegerin inhibits the development of osteolytic bone disease in multiple myeloma. Blood 2001; 98(13): 3534-40.

18. Brown JM, Corey E, Lee ZD et al. Osteoprotegerin and rank ligand expression in prostate cancer. Urology 2001; 57(4): 611-6.

19. Morony S, Capparelli C, Sarosi I et al. Osteoprotegerin inhibits osteolysis and decreases skeletal tumor burden in syngeneic and nude mouse models of experimental bone metastasis. Cancer Res 2001; 61(11): 4432-6.

20. Zhang J, Dai J, Qi Y et al. Osteoprotegerin inhibits prostate cancer-induced osteoclastogenesis and prevents prostate tumor growth in the bone. J Clin Invest 2001; 107(10): 1235-44. 
21. Yonou H, Kanomata N, Goya M et al. Osteoprotegerin/Osteoclastogenesis inhibitory factor decreases human prostate cancer burden in human adult bone implanted into nonobese diabetic/ severe combined immunodeficient mice. Cancer Res 2003; 63(9): 2096-102.

22. Bladou F, Vessella RL, Buhler KR et al. Cell proliferation and apoptosis during prostatic tumor xenograft involution and regrowth after castration. Int J Cancer 1996; 67: 785-90.

23. Ellis WJ, Vessella RL, Buhler KR et al. Characterization of a novel androgen-sensitive, prostate-specific antigen-producing prostatic carcinoma xenograft: LuCaP 23. Clin Cancer Res 1996; 2(6): 1039-48.

24. Corey E, Quinn JE, Bladou F et al. Establishment and characterization of osseous prostate cancer models: Intra-tibial injection of human prostate cancer cells. Prostate 2002; 52(1): 20-33.

25. Corey E, Brown LG, Quinn JE et al. Zoledronic acid exhibits inhibitory effects on osteoblastic and osteolytic metastases of prostate cancer. Clin Cancer Res 2003; 9: 295-306.

26. Goltzman D, Karaplis AC, Kremer R et al. Molecular basis of the spectrum of skeletal complications of neoplasia. Cancer 2000; 88(12 Suppl): 2903-8.

27. Theill LE, Boyle WJ, Penninger JM. RANK-L and RANK: T cells, bone loss, and mammalian evolution. Annu Rev Immunol 2002; 20: 795-823.

28. Lee YP, Schwarz EM, Davies M et al. Use of zoledronate to treat osteoblastic versus osteolytic lesions in a severe-combined-immunodeficient mouse model. Cancer Res 2002; 62(19): 5564-70. 